\title{
Buchbesprechung
}

\section{Kammigan, Ilka: »It's all about interactions«. Eine empirische Untersuchung von Schulgewalt, Normorientierungen und Kontrollen aus Sicht der Situational Action Theory}

Wissenschaftlicher Verlag Berlin 2017, 281 Seiten, € 46,--

https://doi.org/10.1515/mks-2020-2032

Besprochen von Helmut Hirtenlehner: Zentrum für Kriminologie, Johannes Kepler Universität Linz, Altenberger Straße 69, 4040 Linz/ Österreich, E-Mail: helmut.hirtenlehner@jku.at

«It's all about interactions « - der Titel der von Ilka Kammigan als Dissertationsschrift an der Universität Hamburg vorgelegten empirischen Studie zum Zusammenspiel von individuellen und kollektiven Normorientierungen bei der Hervorbringung von Gewalthandlungen in Schulen ist zugleich Programm. Basierend auf einer Befragung von rund 3.000 Schülern der Jahrgangsstufen sieben und neun in Hamburger allgemeinbildenden Schulen wird untersucht, wie persönliche Gewaltbefürwortung und Merkmale des Schulumfeldes - namentlich die im institutionellen Kontext geltenden gewaltbezogenen Verhaltensregeln und das Potenzial der Lehrerschaft zur Durchsetzung der institutionellen Regeln - bei der Erklärung von Schulgewalt zusammenwirken. Als forschungsleitender Bezugsrahmen fungiert Per-Olof Wikströms Situational Action Theory (SAT). Diese besagt im Kern, dass im Zuge einer PersonUmwelt-Interaktion Motivation entsteht, die einen Wahrnehmungs-Entscheidungs-Prozess anstößt, der dann unmittelbar das Handeln steuert. Wahrnehmung - ein zutiefst moralisches Unterfangen - bezieht sich dabei auf das In-Betracht-Ziehen von Handlungsalternativen für die Befriedigung eines Bedürfnisses. Entscheidung beschreibt die Auswahl der subjektiv besten Handlungsoption aus dem perzipierten Alternativenraum.

Die hier zu besprechende Monographie richtet das Hauptaugenmerk auf die Wirkungsdynamik des moralischen Filters und streift das Konzept der bedingten Relevanz von Kontrolle. Ob, um einer bestimmten Motivation Genüge $\mathrm{zu}$ tun, Kriminalität als Handlungsalternative überhaupt in Erwägung gezogen wird, bestimmt sich der Theorie zufolge nach den Moralvorstellungen der Person und dem moralischen Kontext des Settings. Kontrollen be- einflussen die Wahl der zu implementierenden Handlungsweise, sollen aber nur Wirkung entfalten, wenn der moralische Filter versagt hat und Kriminalität in den Bereich der ernsthaft erwogenen Handlungsalternativen vordringen konnte. Letzteres soll vor allem dann der Fall sein, wenn die eigenen moralischen Überzeugungen und die normative Regulierung des Settings im Widerspruch zueinander stehen.

Im empirischen Teil der Arbeit wird detailliert beleuchtet, wie persönliche Moralität (individuelle Normorientierung) und moralischer Kontext (die im schulischen Umfeld dominierenden Normorientierungen) interagieren - sowohl im Hinblick auf die Wahrnehmung von Gewalt als vertretbare Handlungsoption als auch im Hinblick auf das Ausmaß praktizierter Gewalt - und welche modifizierende Bedeutung externaler Kontrolle in der Schule zukommt. Dabei wird ein komplexer (und komplizierter) Hypothesenkatalog entwickelt, der elaborierte Auswertungen im Kleid nicht-linearer Mehrebenenanalysen anleitet, welche sich bei Bedarf noch mit der Berechnung marginaler (Wechselwirkungs-)Effekte schmücken.

Das von der Verfasserin formulierte Hypothesensystem weicht in zwei bemerkenswerten Punkten von der ursprünglichen Theorie ab. Beide Argumente laufen auf eine tendenzielle Aufweichung der von Wikström vorgeschlagenen stringenten Trennung der beiden Phasen des sequenziellen Wahrnehmungs-Entscheidungs-Prozesses hinaus.

1. Handlungsmotivation wird als Ergebnis des moralischen Filters konzeptualisiert.

Unter Handlungsmotivation versteht Kammigan den Willen, ein Ziel mit einer bestimmten Handlungsalternative zu verfolgen. Der Wunsch bzw. die Bereitschaft, ein vorhandenes Bedürfnis mittels Kriminalität zu befriedigen, wird mit der Wahrnehmung von Kriminalität als tauglicher Handlungsoption gleichgesetzt. Konsistent mit diesem konzeptionellen Zugang wird Handlungsmotivation als gewaltbezogene Handlungsintention operationalisiert. 
Kritisch einwenden ließe sich gegen diese Auffassung allerdings, dass damit absichtsbildende Elemente des Auswahlprozesses in die Wahrnehmungsstufe vorgezogen werden.

2. Externale Kontrollen bzw. Kontrolleure beeinflussen aufgrund ihrer normverdeutlichenden Wirkung auch die Wahrnehmung von Handlungsalternativen.

Wenn Kontrollpersonen bzw. Überwachungsinstrumente die in einem Setting geltenden Verhaltensregeln sichtbar machen, rufen sie diese laut Kammigan den Akteuren auch in Erinnerung, was nicht ohne Folgewirkungen auf die moralische Filterung der denkbaren Verhaltensoptionen bleiben sollte. Ferner werden Kontrolleure als im Setting anwesende Personen den moralischen Kontext des sinnlich erfahrbaren Umweltausschnitts mitgestalten, was ihnen eine zusätzliche wahrnehmungsformende Kraft verleiht.

Der Ergebnisteil der Arbeit präsentiert sich etwas sperrig und kompliziert. Aber wie die Autorin (S. 241) selbst anmerkt: »Die Komplexität der Ausgangsannahmen und die Vielzahl der geprüften Hypothesen und Interaktionen machte es möglicherweise nicht ganz einfach, den Erträgen (...) zu folgen.« Es werden eine Fülle von anspruchsvollen Analysen zu insgesamt 14 Hypothesen in sehr differenzierter Form vorgestellt, was die Verdichtung der Befunde auf ihre Implikationen für die forschungsleitenden Fragestellungen doch ein wenig erschwert.

Als Kernergebnis lässt sich identifizieren, dass die in der SAT formulierte Wirkungsdynamik des moralischen Filters durchaus empirische Entsprechung findet, wenn man wie von Kammigan angeregt auf die Entstehung der
Handlungsmotivation (stellvertretend für die Wahrnehmung von Kriminalität als echte Alternative) abhebt, nicht aber, wenn man tatsächliches Gewalthandeln in der Schule zur Zielvariable macht. Letzteres erscheint angesichts des Umstandes, dass die Effekte der moralischen Filterung potenzieller Handlungsalternativen auf das faktische Handeln durch Kontrollen modifiziert werden, durchaus theoriekompatibel. Im Einklang mit den theoretischen Erwartungen steht auch die Beobachtung, dass das perzipierte Kontrollpotenzial des schulischen Umfeldes dort seinen größten Erklärungsbeitrag entfaltet, wo die persönliche Gewaltbefürwortung hoch ist. Die überformende Rolle schulischer Kontrollen impliziert dabei, dass Handlungsintentionen «nicht allein aus methodischen Gründen als Ersatz für die Messung tatsächlichen delinquenten Verhaltens verwendet werden sollte[n]«.

Alles in allem legt Ilka Kammigan mit ihrer Dissertationsschrift ein gut recherchiertes und handwerklich ansprechendes Werk vor, das sich seinen Platz in der Bibliothek der Evidenzen zur SAT redlich verdient hat. Selten wurden ähnlich detaillierte Analysen zur Wirkungsdynamik des moralischen Filters vorgenommen. Was allerdings ob der dezidierten Hinwendung auch zum Konzept der bedingten Relevanz von Kontrolle etwas erstaunt, ist die Ausblendung einer möglichen Wechselwirkung zwischen dem normativen Schulumfeld und der individuellen Selbstkontrolle. Da im verwendeten Datensatz für beide Konstrukte Operationalisierungen verfügbar sind, verwundert es ein wenig, dass das Zusammenspiel der Fähigkeit zur Ausübung von Selbstkontrolle mit dem moralischen Kontext nicht eine zur Abschreckungs-Gewaltbefürwortungs-Interaktion analoge Bedeutung erhielt. 